\title{
Cholesterol Synthesis Is Required for Cutaneous Barrier Function in Mice
}

Kenneth R. Feingold, Man Mao-Qiang, Gopinathan K. Menon, Song S. Cho, Barbara E. Brown, and Peter M. Elias

Dermatology and Medical Services, Veterans Administration Medical Center; and the Departments of Dermatology and Medicine,

University of California School of Medicine, San Francisco, California 94121

\begin{abstract}
Previous studies have shown that topical acetone treatment results in the removal of stratum corneum lipids and disruption of the permeability barrier. This disruption stimulates epidermal lipid synthesis which is associated with the rapid restoration of stratum corneum lipids and barrier function. The aim of this study was to determine the role of cutaneous cholesterol synthesis in the barrier recovery. Here we show that topical lovastatin, a competitive inhibitor of HMG CoA reductase, inhibits cholesterol synthesis. After acetone disruption of the barrier, the normal rapid return of cholesterol to the stratum corneum and recovery of barrier function is impaired in animals treated topically with lovastatin. When lovastatin animals are simultaneously treated topically with either mevalonate, the immediate product of HMG CoA reductase, or cholesterol, the final end product of the pathway, the recovery of the barrier is normalized. Lovastatin resulted in the delayed secretion and abnormal appearance of lamellar bodies. These results provide the first evidence demonstrating that cholesterol synthesis is required for the maintenance of barrier structure and function and suggests a crucial role for cholesterol synthesis in allowing for terrestrial existence. (J. Clin. Invest. 1990. 86:1738-1745.) Key words: lovastatin • epidermis - lipid synthesis - stratum corneum • transepidermal water loss
\end{abstract}

\section{Introduction}

The major function of the epidermis is to form a protective layer, the stratum corneum, which prevents the excessive loss of bodily fluids (1). Lipids account for only a small percentage of total stratum corneum weight, but are crucial for the permeability barrier $(2,3)$. The epidermis is a very active site of both sterol and fatty acid synthesis, with most of the lipids that account for the cutaneous barrier synthesized in the epidermis itself rather than deriving from extracutaneous sites (4-8).

Epidermal sterol synthesis appears to be relatively autonomous from systemic influences $(5,9)$, presumably due to the paucity of lipoprotein receptors on the membranes of these cells (10-13). In contrast, studies have shown that perturbations in permeability barrier function greatly influence epidermal lipid synthesis (14-16). When the cutaneous permeability

Address correspondence and reprint requests to Kenneth R. Feingold, Metabolism Section (111F), VA Medical Center, 4150 Clement Street, San Francisco, CA 94121.

Received for publication 7 November 1989 and in revised form 18 May 1990.

J. Clin. Invest.

(c) The American Society for Clinical Investigation, Inc.

$0021-9738 / 90 / 11 / 1738 / 08 \$ 2.00$

Volume 86, November 1990, 1738-1745 barrier is disrupted by topical treatment with either solvents or detergents, a marked stimulation of both epidermal sterol and fatty acid synthesis occurs, which returns to normal in parallel with barrier recovery $(14,15)$. Moreover, essential fatty aciddeficient mice, which exhibit a dietarily induced disturbance in barrier function, also display increased epidermal lipid synthesis (16). Furthermore, when the defect in barrier function, in all three models, is artifically corrected by occlusion with a water vapor-impermeable membrane, no increase in epidermal sterol and fatty acid synthesis is observed (14-16). In contrast, occlusion with water vapor-permeable membranes did not prevent the expected increase in epidermal lipid synthesis (17). These observations suggest that water flux through the epidermis may be a crucial factor in the regulation of epidermal lipid synthesis.

That this regulation of epidermal lipid synthesis by barrier function has physiological implications has been demonstrated. Topical solvent treatment results in the removal of stratum corneum lipids, which leads to a marked increase in transepidermal water loss $(14,15,17)$. Over 24 to $48 \mathrm{~h}$, the lipid content of the stratum corneum returns to control levels, and in association with the return of stratum corneum lipids, barrier function returns to normal (17). If, after disrupting the barrier, animals are covered with a water-impermeable membrane that restores barrier function and prevents the increase in epidermal lipid synthesis, the return of stratum corneum lipids is prevented (17). Additionally, the disturbance in barrier function remains equal to or greater than that observed immediately after acetone treatment. In contrast, when acetone-treated animals are covered with a water vapor-permeable membrane, which does not prevent the usual increase in epidermal lipid synthesis, both stratum corneum lipid content and barrier function return towards normal (17). These observations suggest that the stimulation of epidermal lipid synthesis after barrier perturbation plays an important role in both the restoration of stratum corneum lipid content and the recovery of barrier function.

The above studies point to a central role for cutaneous lipid synthesis. The aim of this study was to determine the specific role of cutaneous cholesterol synthesis in the recovery of barrier structure and function after disruption of the permeability barrier with solvent treatment. In these experiments, we have specifically determined the effect on barrier function of inhibiting cutaneous cholesterol synthesis by the topical administration of lovastatin, a drug that competitively inhibits HMG CoA reductase, the rate limiting enzyme in cholesterol synthesis (18).

\section{Methods}

\section{Materials}

Hairless male mice (hr/hr) 8-10 wk old were purchased from Jackson Laboratories (Bar Harbor, ME). They were fed Simonsen mouse diet (Gilroy, CA) and water ad lib. The age ranged between 10 to $12 \mathrm{wk}$ at 
Table I. Effect of Topical Lovastatin Treatment on Epidermal and Dermal Lipid Synthesis

\begin{tabular}{|c|c|c|c|c|}
\hline & \multicolumn{2}{|c|}{ Epidermis } & \multicolumn{2}{|c|}{ Dermis } \\
\hline & Cholesterol & Fatty acids & Cholesterol & Fatty acids \\
\hline & \multicolumn{2}{|c|}{ nmol incorporated/g per $h$} & \multicolumn{2}{|c|}{ nmol incorporated/g per $h$} \\
\hline \multicolumn{5}{|l|}{$1 \mathrm{~h}$} \\
\hline Control $(n=4)$ & $0.562 \pm 0.105$ & $5.53 \pm 0.36$ & $0.133 \pm 0.022$ & $1.33 \pm 0.12$ \\
\hline \multirow{2}{*}{ Lovastatin $(n=4)$} & $0.069 \pm 0.009$ & $5.53 \pm 0.74$ & $0.017 \pm 0.003$ & $1.32 \pm 0.23$ \\
\hline & $P<0.01$ & NS & $P<0.01$ & NS \\
\hline \multicolumn{5}{|l|}{$3 \mathrm{~h}$} \\
\hline Control $(n=6)$ & $0.581 \pm 0.144$ & $3.87 \pm 0.80$ & $0.089 \pm 0.018$ & $0.319 \pm 0.113$ \\
\hline \multirow[t]{2}{*}{ Lovastatin $(n=6)$} & $0.177 \pm 0.023$ & $3.44 \pm 0.29$ & $0.049 \pm 0.017$ & $0.253 \pm 0.042$ \\
\hline & $P<0.02$ & NS & NS & NS \\
\hline
\end{tabular}

Hairless mice were treated topically on one flank with either lovastatin or vehicle. At 1 or $3 \mathrm{~h}$ after topical treatment, the animals were killed and the full thickness skin samples were incubated in a Krebs phosphate buffer containing $\left[{ }^{14} \mathrm{C}\right]$ acetate for $1 \mathrm{~h}$ at $37^{\circ} \mathrm{C}$. At the end of the incubation, the epidermis and dermis were separated and individually saponified in a $\mathrm{KOH}$ ethanol solution. The incorporation of $\left[{ }^{14} \mathrm{C}\right] \mathrm{acetate}$ was determined after petroleum ether extraction as described in Methods. Values are mean \pm SE.

the time of study. Acetone was purchased from Fisher Scientific Co. (Fairlane, NJ). 1 [ $\left.{ }^{14} \mathrm{C}\right]$ acetate $(45-60 \mathrm{mCi} / \mathrm{mmol}),\left[{ }^{3} \mathrm{H}\right]$ cholesterol $(40-60 \mathrm{Ci} / \mathrm{mmol}), 26\left[{ }^{14} \mathrm{C}\right]$ cholesterol $(0.5 \mathrm{mCi} / 0.33 \mathrm{mg})$, and $\left[{ }^{3} \mathrm{H}\right]-$ oleic acid $(2-10 \mathrm{Ci} / \mathrm{mmol})$ were purchased from New England Nuclear (Boston, MA). Tritiated water ( $1 \mathrm{Ci} / \mathrm{g}$ ) was purchased from ICN Biochemicals (Costa Mesa, CA). Nile red and filipin were purchased from Polyscience Inc. (Warrington, PA). Cholesterol, mevalonate, and lipid standards were purchased from Sigma Chemical Co. (St. Louis, MO). Lovastatin was kindly provided by Dr. A. Alberts of Merck, Sharp, and Dohme. High performance thin layer chromatography plates (HPTLC Silica Gel 60) were obtained from E. Merck (Darmstadt, FRG). The lovastatin was prepared by incubating in a $0.1 \mathrm{~N} \mathrm{KOH}$ solution for $2 \mathrm{~h}$ at $50^{\circ} \mathrm{C}$ after which the solution was neutralized to $\mathrm{pH} 7.4$ with $\mathrm{HCl}$.

\section{Experimental procedures}

Solvent treatment and water loss measurements. To acutely perturb barrier function, the flanks of hairless mice were gently treated with acetone-soaked cotton balls as described in previous publications (14, $15,17)$. Acetone treatment does not result in visible or microscopic damage to the stratum corneum. After acetone treatment, one flank $\left(\sim 2 \mathrm{~cm}^{2}\right)$ was treated topically with $30 \mu \mathrm{l}$ of a polypropylene glycol/ ethanol solution $(7: 3 \mathrm{vol})$ containing $25 \mathrm{mg}$ of lovastatin per milliliter, while the other flank was treated with vehicle alone and served as a control. In some experiments the polypropylene glycol/ethanol solution contained either mevalonate $(25 \mathrm{mg} / \mathrm{ml})$ or cholesterol $(25$ $\mathrm{mg} / \mathrm{ml}$ ) in addition to lovastatin.

Immediately after acetone treatment, transepidermal water loss (TEWL) ${ }^{1}$ was measured using a Meeco electrolytic analyzer, as described in previous publications (14-17). Animals with TEWL rates between 250 and $900 \mathrm{ppm} / 0.5 \mathrm{~cm}^{2}$ per h $\left(<20 \mathrm{ppm} / 0.5 \mathrm{~cm}^{2}\right.$ per $\mathrm{h}$ is normal) were included in the study. Immediately after the measurement of TEWL, animals were treated topically with either the vehicle, lovastatin, lovastatin plus mevalonate, or lovastatin plus cholesterol and the TEWL loss was measured at hourly intervals thereafter.

Histochemical staining. Nile red, a fluorescent probe for lipids, was used to demonstrate the distribution and content of lipid in the stratum corneum as described previously (17). Filipin is a macrolide, antifungal antibiotic that forms a one to one stoichiometric complex with free 3B-OH sterols, and has proven to be a useful probe for cholesterol-enriched structures (19). An aqueous solution containing

1. Abbreviations used in this paper: SC, stratum corneum; SG, stratum granulosum; TEWL, transepidermal water loss.
$150 \mu \mathrm{M}$ filipin was applied to fresh frozen sections, and the cholesterol-enriched sites in the epidermis were detected by fluorescence microscopy at $400 \mathrm{~nm}$ excitation and $500 \mathrm{~nm}$ emission frequency (20).

Electron microscopy. At various time points after acetone disruption of the barrier skin biopsies were taken for electron microscopy. The samples were minced to $0.5 \mathrm{~mm}$ and fixed in half-strength Karnovsky's fixative overnight, washed in cacodylate buffer, and postfixed in $1 \%$ osmium tetroxide-containing $1.5 \%$ potassium ferrocyanide. After fixation, samples were dehydrated in graded ethanol solutions and embedded in an Epon-epoxy mixture (20). 600-800-nm sections were double strained with uranyl acetate and lead citrate and examined in an electron microscope (model 10A; Carl Zeiss, Inc., Thornwood, $\mathrm{NY}$ ) operated at $60 \mathrm{kV}$.

Lipid analysis of stratum corneum. Stratum corneum sheets were obtained from hairless mice by tape stripping of a specified area as described previously (21). The stratum corneum, which adhered to the tape, was then emersed in water for $1 \mathrm{~h}$ to separate the plastic backing from the gum and stratum corneum. The stratum corneum lipids were extracted by the method of Bligh and Dyer (22), dried, and suspended in chloroform for thin layer chromatography. The entire lipid extract and standards were applied to precleaned TLC plates and fractionated in a neutral lipid (petroleum ether/diethyl ether/glacial acetic acid, 80:20:1 vol) solvent system. The major species were identified under ultraviolet-A (UV-A) fluorescence after spraying with $0.25 \%$ aqueous 8-anilino-1-naphthalene sulfonic acid, scraped off the plates, and extracted with Bligh-Dyer solvents, and the cholesterol was quantitated spectrophotometrically by the method of Ham et al. (23). Cholesterol content was normalized to tissue area.

Lipid synthesis (in vitro studies). 1 and $3 \mathrm{~h}$ after topical treatment with either lovastatin or vehicle, full thickness skin samples were removed, the fat was scraped off, and the samples were weighed and placed dermis downward in $2 \mathrm{ml}$ of a Krebs phosphate-buffered saline (pH 7.4) (calcium, magnesium free) containing $40 \mu \mathrm{Ci}$ of $\left[{ }^{14} \mathrm{C}\right]$ acetate and $10 \mathrm{mM}$ EDTA. The full thickness skin samples were incubated for $1 \mathrm{~h}$ at $37^{\circ} \mathrm{C}$ and then the epidermis was separated from the dermis. The epidermis and dermis from each example were then saponified individually in a solution of $45 \% \mathrm{KOH}$, water, and $70 \%$ ethanol (2:1:5) overnight at $42^{\circ} \mathrm{C}$. After adding internal standards of tritiated cholesterol and tritiated oleic acid, the nonsaponifiable lipids were extracted with petroleum ether, dried, dissolved in chloroform, and then applied to TLC plates. The band corresponding to a standard of cholesterol was scraped from the plate and counted by liquid scintillation spectrometry. Fatty acids were extracted with petroleum ether after acidification of the remaining saponification fluid. The material was dried 


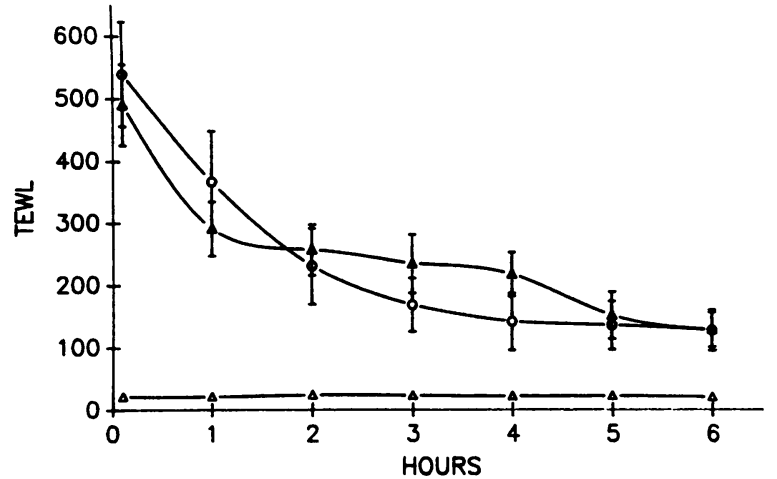

Figure 1. Barrier function after acetone disruption. Hairless mice were treated topically with either saline alone $(-\Delta-)(n=5)$, acetone alone $(-\Delta-)(n=9)$, or acetone followed by vehicle (polypropylene glycol/ethanol, 7:3 vol) $(-\circ-)(n=7)$. TEWL was measured hourly as described in Methods. The data are expressed as mean \pm SE.

and dissolved in chloroform, and an aliquot was counted as described above. Calculations were corrected for their respected spillovers, background, and recovery of internal standards.

Lipid synthesis (in vivo studies). The incorporation of tritiated water into cholesterol in the epidermis and dermis was determined as described previously $(8,14-16)$.

The statistical significance was determined using the Student's $t$ test.

\section{Results}

Lipid synthesis. Table I. shows the effect of topical lovastatin treatment on the in vitro incorporation of acetate into cholesterol and fatty acids in the epidermis and dermis. Between 1 and $2 \mathrm{~h}$ after topical lovastatin, cholesterol synthesis is decreased by $88 \%$ in the epidermis and by $87 \%$ in the dermis. At 3-4 $\mathrm{h}$ after lovastatin treatment, cholesterol synthesis is decreased by $70 \%$ in the epidermis and by $45 \%$ in the dermis. As expected, at both time periods lovastatin does not affect either epidermal or dermal fatty acid synthesis. Similar effects of lovastatin treatment were observed after acetone disruption of the barrier. The in vivo incorporation of tritiated water into cholesterol from 1 to $4 \mathrm{~h}$ after acetone and lovastatin treatment is inhibited $56 \%$ in the epidermis (control, 0.502 vs. lovastatin, $0.223 \mu \mathrm{mol} / 3 \mathrm{~h}$ per $\mathrm{g}$ ) and $41 \%$ (control, 0.976 vs. lovastatin, $0.576 \mu \mathrm{mol} / 3 \mathrm{~h}$ per $\mathrm{g}$, mean of two animals) in the dermis in comparison to animals treated with acetone and

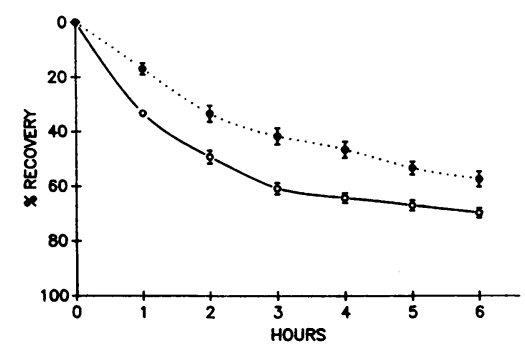

Figure 2. Effect of lovastatin on barrier recovery. Hairless mice were treated topically with acetone to disrupt the barrier, followed by either lovastatin $(\cdot \bullet \cdot)$ or vehicle alone $(-0-)$. TEWL was measured hourly as described in Methods. The data are presented as the percent recovery of TEWL and are expressed as mean \pm SE. $n=45$ for control, $n=50$ for lovastatin, $P<0.001$ for all time points.

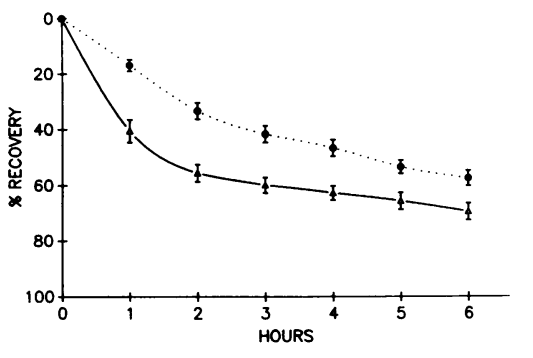

Figure 3. Effect of mevalonate on the lovastatin inhibition of barrier recovery. Hairless mice were treated topically with acetone to disrupt the barrier, followed by either lovastatin alone $(\cdot \bullet \cdot)$ or lovastatin plus mevalonate $(-\Delta-)$.

TEWL was measured

hourly as described in Methods. The data are presented as the percent recovery of TEWL and are expressed as mean \pm SE. $n=50$ for lovastatin alone, $n=15$ for lovastatin plus mevalonate. $P<0.001$ for 1-, 2-, 3-, and 4-h time points; $P<0.01$ for 5- and 6-h time points.

saline. Thus, topical lovastatin treatment inhibits cholesterol synthesis in both the epidermis and dermis.

Transepidermal water loss. As described previously, topical acetone disrupts barrier function and increases transepidermal water loss. This disruption is followed by a rapid recovery with transepidermal water loss rates decreasing by $65-80 \%$ over a 6-h period (Fig. 1). This rapid recovery is not altered by vehicle treatment. As expected, animals treated topically with saline alone do not have a defect in barrier function.

The effect of topical lovastatin on recovery of barrier function after acetone treatment is shown in Fig. 2. When acetone disruption of the barrier is followed by topical lovastatin, the recovery of barrier function is slowed and the percentage recovery is decreased significantly at all time points. At early time periods, lovastatin treatment results in a $\sim 50 \%$ inhibition in barrier recovery, whereas at later time points the inhibition is less. These results indicate that topical lovastatin, which inhibits cutaneous cholesterol synthesis, delays the recovery of barrier function after acetone treatment.

To determine whether the effects of lovastatin on barrier recovery are specifically due to an inhibition of HMG CoA reductase activity, we next compared the recovery of transepidermal water loss in lovastatin animals with animals simultaneously treated with lovastatin plus mevalonic acid, the immediate product of the reaction catalyzed by HMG CoA reductase. As shown in Fig. 3, barrier recovery is restored to normal when lovastatin animals are simultaneously treated with mevalonate. This indicates that the lovastatin-induced delay in barrier recovery is due to inhibition of HMG CoA reductase activity.

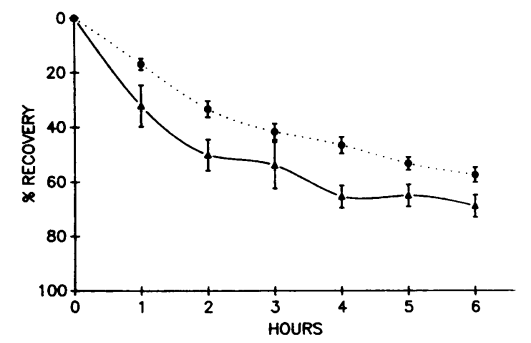

Figure 4. Effect of cholesterol on the lovastatin inhibition of barrier recovery. Hairless mice were treated topically with acetone to disrupt the barrier, followed by either lovastatin alone $(\cdot \bullet \cdot)$ or lovastatin plus cholesterol $(-\Delta-)$.

TEWL was measured as described in Methods. The data are presented as the percent recovery of TEWL and are expressed as mean $\pm \mathrm{SE} n=\mathbf{5 0}$ for lovastatin alone, $n=10$ for lovastatin plus cholesterol. $P<0.05$ for 1 - and 6 -h time points; $P<0.02$ for 2 - and 5 -h time points; $P<0.001$ for the $4-\mathrm{h}$ time point. 
Table II. Summary of Fluorescence Results

\begin{tabular}{|c|c|c|c|c|}
\hline & \multirow[b]{2}{*}{ Pretreatment } & \multicolumn{3}{|c|}{$\begin{array}{l}\text { Time after acetone } \\
\text { treatment }(\mathrm{h})\end{array}$} \\
\hline & & $0-1$ & $3-4$ & 6 \\
\hline \multicolumn{5}{|l|}{ Nile red } \\
\hline Acetone + vehicle & ++++ & 0 & ++ & +++ \\
\hline Acetone + lovastatin & ++++ & 0 & ++ & +++ \\
\hline \multicolumn{5}{|l|}{ Filipin } \\
\hline Acetone + vehicle & ++++ & 0 & ++ & +++ \\
\hline $\begin{array}{c}\text { Acetone + lovastatin } \\
\text { Scale: } 0-4+\text { (maximum) }\end{array}$ & ++++ & 0 & $+1-$ & + \\
\hline
\end{tabular}

Hairless mice were treated topically with acetone to disrupt the barrier, followed by application of either lovastatin or vehicle alone. At the times indicated after treatment, skin biopsies were obtained and fresh frozen sections were stained with either Nile red or filipin as described in Methods. Intensity ranges from 0 (nonobserved) to 4+ (maximum fluorescence for all samples); ratings are semiquantitative and based upon both visual assessment and length of photographic exposure.

In addition to cholesterol, mevalonate can be converted to a number of other potentially important end products. Therefore, to determine whether inhibition of cholesterol synthesis by lovastatin is the mechanism that accounts for the delay in barrier recovery, we compared lovastatin animals with animals treated simultaneously with lovastatin plus cholesterol. As shown in Fig. 4; the rate of barrier recovery is normalized when lovastatin animals are treated simultaneously with topical cholesterol. This indicates that the lovastatin-induced delay in barrier recovery is due to an inhibition of cutaneous cholesterol synthesis.

Histochemical staining. As described in detail in previous studies, immediately after acetone treatment there is an absence of nile red-stainable lipids in the stratum corneum (17). Over time nile red staining reveals the return of bright yellow fluorescence in the stratum corneum, indicative of the return of neutral lipids. As shown in Table II, the pattern of recovery of nile red staining after acetone treatment is similar with or without lovastatin treatment.

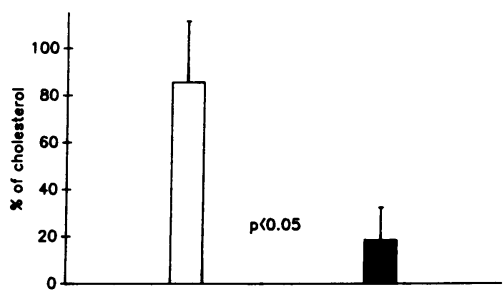

Figure 6. Recovery of cholesterol in the stratum corneum. Animals whose barrier was disrupted with acetone and treated with vehicle alone ( $\square$ ) were compared with animals whose barrier was

disrupted with acetone and treated with lovastatin (ø). Stratum corneum sheets were isolated $3 \mathrm{~h}$ after barrier disruption and the quantity of cholesterol determined as described in Methods. Data are expressed as percent recovery of cholesterol and presented as the mean \pm SE. $n=3$ for both groups.

When normal skin sections are stained with filipin, a specific fluorescence probe for free 3-B-hydroxysterols (19), the stratum corneum fluoresces green, indicative of enrichment in cholesterol (20). Immediately after acetone treatment, filipin staining is virtually absent in the stratum corneum, indicating that acetone treatment removes substantial amounts of cholesterol from the stratum corneum. Over time the filipinstainable lipid returns to the stratum corneum at rates similar to those observed using nile red as the histochemical probe (Fig. 5, $A$ and $B$ ). However, when acetone-treated animals are treated topically with lovastatin, the return of filipin staining to the stratum corneum is slowed (Fig. $5 C$, Table II). These results indicate that after acetone extraction of stratum corneum lipids, restoration of stratum corneum lipids occurs over time in controls. In contrast, the lovastatin-induced inhibition of cutaneous cholesterol synthesis results in a specific delay in the reaccumulation of histochemically stainable cholesterol in the stratum corneum without delaying the overall return of other neutral lipids.

Stratum corneum lipid analysis. To confirm the histochemical observations, we next compared the recovery of cholesterol in stratum corneum topically treated with acetone $3 \mathrm{~h}$ earlier and stratum corneum treated with acetone plus lovastatin. As illustrated in Fig. 6, the return of cholesterol to the stratum corneum is markedly delayed in the lovastatin-treated animals. These results confirm the histochemical staining studies and further demonstrate that lovastatin inhibition of cutaneous cholesterol synthesis delays the return of cholesterol to the stratum corneum.
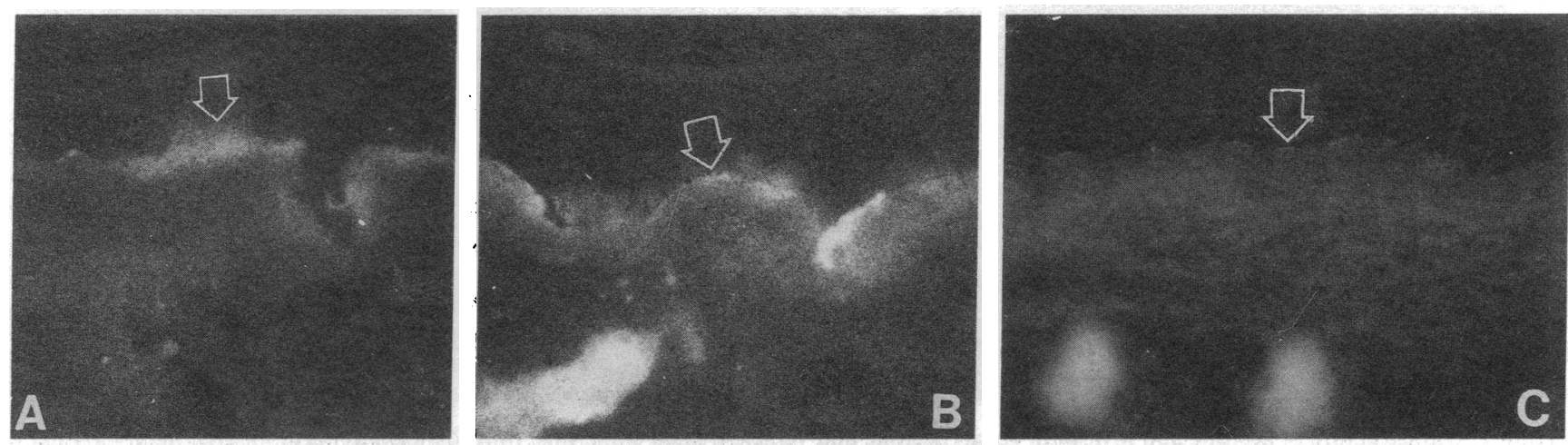

Figure 5. Frozen sections of hairless mouse skin stained with filipin after acetone treatment. Whereas immediately after acetone treatment no fluorescence can be detected (not shown), by $3(A)$ and $6(B) \mathrm{h}$ considerable reaccumulation of filipin-stainable free sterols has occurred (arrows). In contrast, in acetone plus lovastatin-treated animals $(C)$, even after $6 \mathrm{~h}$ there is a substantial delay in the reaccumulation of cholesterol (arrow in $C$ points to a comparable site in $B$; c.f. Table II). A-C, $\times 750$. 

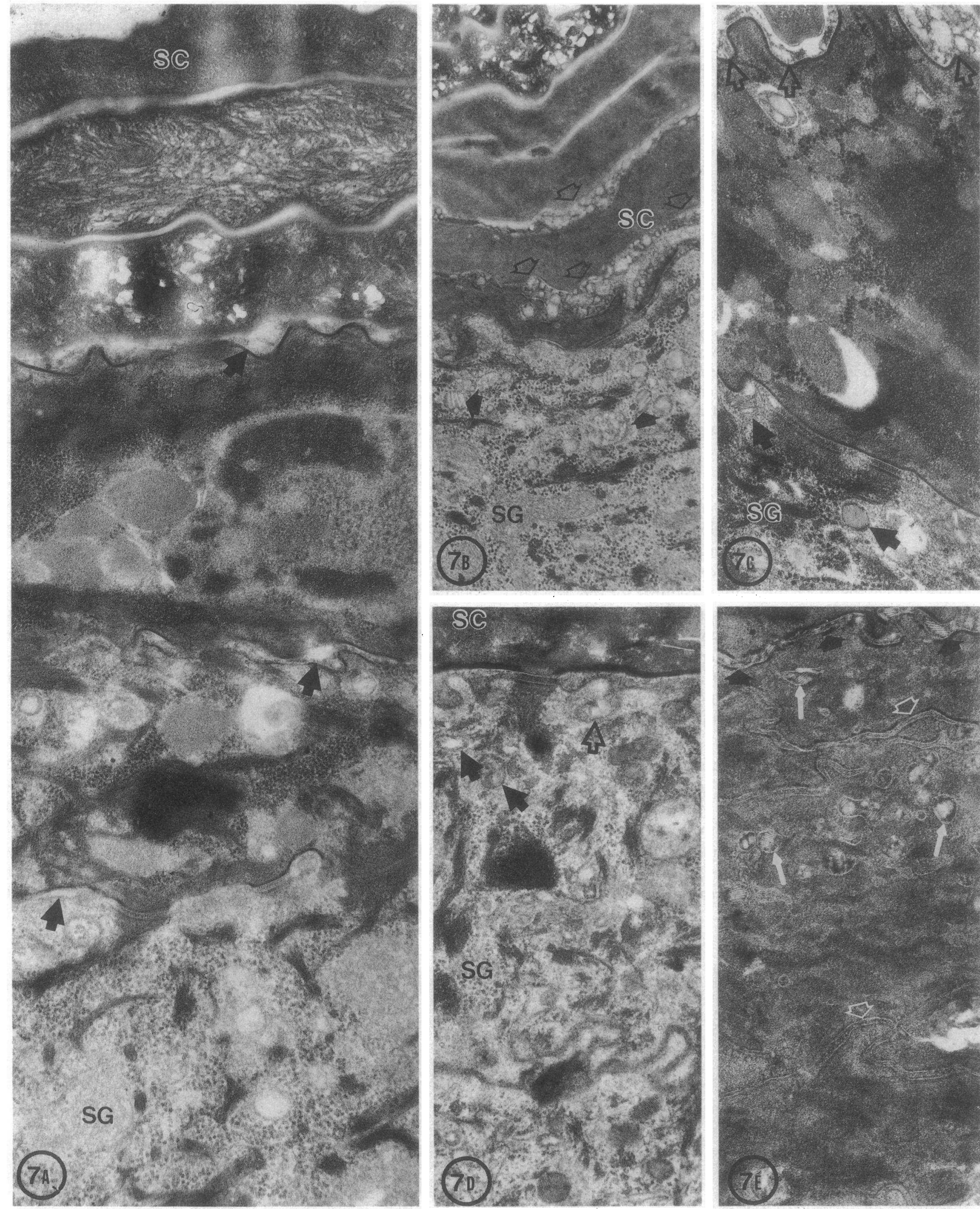

Figure 7. Ultrastructure of acetone and lovastatin versus acetone and vehicle-treated epidermis. Immediately after acetone treatment $(A)$ the cytoplasm of the stratum granulosum $(S G)$ is devoid of lamellar bodies. Moreover, the intercellular spaces of the stratum corneum ( $S C$ ) also contain a paucity of lamellar body-derived material (arrows), presumably due to removal by repeated acetone treatments. By 1 and $3 \mathrm{~h}$ after acetone and vehicle treatment ( $B$ and $C$, respectively), numerous lamellar bodies reappear in the cytoplasm of the SG; some of these organelles display normal internal lamellations (closed arrows), and the SC intercellular spaces are replete with newly secreted lamellar body contents (open 
Ultrastructural studies. To ascertain the basis for the lovastatin effect on barrier recovery, we examined skin biopsies immediately after acetone treatment, and 1, 3, and $6 \mathrm{~h}$ after acetone or vehicle applications. As seen in Fig. $7 A$, acetone rapidly depletes the cytosol of virtually all lamellar bodies. Simultaneously, only limited amounts of secreted lamellar body contents remain in the intercellular spaces between the lowermost cornified cells, presumably due to removal during the repeated acetone treatments required to abrogate the barrier, a process that requires $20-30 \mathrm{~min}$. Thus, acetone treatment acutely induces massive exocytosis of lamellar bodies and depletes the intercellular spaces of most lamellar body-derived material.

By $1 \mathrm{~h}$ after acetone, numerous lamellar bodies have reappeared in the cytosol (Fig. $7 \mathrm{~B}$ ). Many of these structures display normal lamellations, and large numbers of these organelles appear to be secreted into intercellular spaces between the lowermost cornified cells, even by $1 \mathrm{~h}$. Likewise, 3 and $6 \mathrm{~h}$ after acetone, large numbers of lamellar bodies are present in the cytosol (many with normal internal lamellations), and continue to be secreted into the intercellular spaces (Fig. $7 C$; 6-h time point not shown).

Lovastatin-treated epidermis at 1,3 , and $6 \mathrm{~h}$ displays abundant lamellar bodies. However, both normal-appearing lamellar bodies and organelles lacking a normal internal structure are present (Fig. $7 \mathrm{D}$ ). Moreover, their secretion also appears to be impaired; i.e., the intercellular spaces of the lower stratum corneum contain extensive regions with a virtual absence of lamellar material, and retained lamellar bodies are present in the cytosol of cornified cells (Fig. 7, $D$ and $E$; 6-h time point not shown). Thus, lovastatin treatment does not block the formation of nascent lamellar bodies, but such treatment induces abnormalities both in the internal structure of these organelles and in the process of lamellar body secretion.

\section{Discussion}

This manuscript provides the first direct evidence that cutaneous cholesterol synthesis is required for the maintenance of permeability barrier structure and function. After disruption of the barrier with topical acetone, the normally rapid recovery of barrier function is impaired in animals treated topically with lovastatin, a specific competitive inhibitor of the enzyme HMG CoA reductase (18). Topical lovastatin results in a marked decrease in cutaneous cholesterol synthesis, and the recovery of barrier function is inhibited initially by $\sim 50 \%$. With time the lovastatin-induced decrease in cutaneous cholesterol synthesis diminishes, and the inhibition of barrier function recovery lessens such that by $6 \mathrm{~h}$ barrier recovery is reduced by $\sim 20 \%$. That the delay in recovery of barrier function in lovastatin animals is due specifically to inhibition of HMG CoA reductase activity is demonstrated by the normalization of barrier recovery that occurs when lovastatin animals are simultaneously treated topically with mevalonate, the immediate product of the enzyme HMG CoA reductase. Moreover, our studies further demonstrate that topically administered cholesterol, the final end product of the sterol biosynthetic pathway, is also capable of reversing the lovastatin-induced inhibition of barrier function recovery. Together these studies provide direct evidence that cutaneous sterol synthesis is required for the immediate recovery of barrier function after acetone treatment.

Of note is that recent studies have demonstrated that inhibition of epidermal sphingolipid synthesis does not inhibit the immediate recovery of barrier function. Using an experimental protocol similar to that employed in our lovastatin studies, we observed that topical treatment with chloroalanine, an inhibitor of sphingolipid synthesis, does not alter the initial recovery of the barrier (24). This indicates that inhibition of the synthesis of other stratum corneum lipids does not necessarily delay the initial recovery of barrier function. Of course, given the very complex biosynthetic and secretory processes required for barrier recovery, it is likely that inhibition of a number of different metabolic pathways could alter barrier recovery.

Studies of the chemical structure of the stratum corneum after barrier disruption provide further insights into the mechanisms accounting for the lovastatin-induced inhibition of barrier recovery. Immediately after topical acetone, histochemical staining of the stratum corneum demonstrates a marked reduction in the staining of neutral lipids and free 3B-OH sterols. Over time, the quantities of both neutral lipids and free 3B-OH sterols increase in the stratum corneum and correlate with barrier function recovery. In contrast, in lovastatin animals the return of stainable free sterols is delayed in the stratum corneum, whereas the return of other neutral lipids appears to be unaffected. Direct biochemical analysis of stratum corneum lipid composition after barrier disruption with acetone demonstrated that the recovery of free sterols in the stratum corneum of lovastatin animals is decreased. Thus, complementary data from both histochemical and biochemical studies demonstrate that lovastatin specifically delays the return of free sterols in the stratum corneum. Furthermore, these results provide strong evidence that sterols in the stratum corneum play an important role in the permeability barrier, a conclusion that we had previously reached from extraction studies that demonstrated that the removal of nonpolar lipids, other than cholesterol and sphingolipids, from the stratum corneum result in only a modest perturbation of barrier function (25).

The electron microscopic studies described in this manuscript suggest mechanisms by which inhibiting epidermal cholesterol synthesis could effect the lipid composition of the stratum corneum. The redeposition of barrier lipids in the intercellular spaces of the stratum corneum occurs via the exocytosis of lipid-rich contents of lamellar bodies $(2,3)$. Immediately after acetone treatment, the cytosol of stratum

arrows). In 1 and $3 \mathrm{~h}$ acetone plus lovastatin-treated animals ( $D$ and $E$, respectively), numerous lamellar bodies appear in the SG. Although many of these organelles appear to possess a normal internal structure, others display an abnormal or empty internal structure (closed arrows). Moreover, less lamellar body-derived material appears to be secreted into the intercellular spaces than in controls ( $E$, open arrows), and as a result extensive areas of the intercellular spares are devoid of lamellar material. Only in occasional foci can some lamellar material be detected $(E$, closed arrows). This process of impaired secretion is more evident at $3 \mathrm{~h}$, where numerous residual lamellar bodies are evident in the cytosol of the lowermost cornified cells $(E$, closed arrows). $A, \times 37,500 ; B, \times 31,250 ; C, \times 40,000 ; D, \times 48,000 ; E, \times 32,000$. 
granulosum cells and the intercellular spaces of the stratum corneum are virtually devoid of lamellar body-derived lipids. Shortly thereafter, however, a large increase in the number of lamellar bodies and their secreted contents occurs and many of those organelles possess normal internal structure. These observations indicate that barrier disruption acutely provokes the exocytosis of preformed lamellar bodies. By $1 \mathrm{~h}$ after acetone disruption of the barrier, nascent lamellar bodies are observed both within the cytosol and in the extracellular spaces. This suggests that the synthesis of "new" lamellar bodies occurs rapidly after barrier disruption. In contrast, in acetone plus lovastatin animals at $\mathbf{1 ~ h}$, many of the intracellular lamellar bodies appear abnormal in structure. As a result, the amount of secreted extracellular lamellar body contents is less than that observed with acetone alone. These observations suggest that lovastatin induces the formation and deposition of nascent lamellar bodies having an abnormal structure, most likely due to an abnormal lipid composition (i.e., decreased cholesterol content). In the acetone plus lovastatin animals, abnormal-appearing lamellar bodies are still seen in both intra- and extracellular locations, but most importantly, secretion appears to be partially impeded. These observations suggest that the altered distribution of lipids in the stratum corneum is due to both the secretion of lamellar bodies containing decreased quantities of cholesterol, and to impairment of the secretory process itself. At the present time it is impossible to definitively prove this hypothesis because techniques to isolate completely homogeneous fractions of pure lamellar bodies have not been developed. Although these results clearly point to the importance of cholesterol for the formation of mammalian permeability barrier, the exact mechanism whereby sterols maintain barrier function remains to be elucidated.

It is likely that both the abnormal appearance of lamellar bodies and the abnormal stratum corneum lipid composition result from lovastatin inhibition of de novo cutaneous cholesterol synthesis, which in turn leads to a deficiency of newly synthesized free sterols for packaging in lamellar bodies. This scheme assumes that, at least acutely, local cutaneous sterol synthesis contributes a significant portion of the sterols required for normal lamellar body formation. Previous studies have demonstrated that large quantities of sterols are synthesized in the skin (4-8). Moreover, disruption of barrier function results in a rapid increase in epidermal sterol synthesis (14-17). Additionally, studies have demonstrated that the delivery of circulating sterols to the epidermis is limited and does not increase after barrier disruption (14-16). In light of these observations, it is logical that inhibition of cutaneous sterol synthesis with topical lovastatin results in the formation of lamellar bodies deficient in sterols and a delay in the return of sterols to the stratum corneum with a resultant inhibition of barrier recovery after disruption of the barrier with acetone. Of course, it should be recognized, as noted above, that preformed lamellar bodies are rapidly secreted after barrier perturbation and may contribute to stratum corneum recovery (2, 3 ). This may account for the relatively modest inhibition of barrier recovery observed in these studies. Additionally, cholesterol stored in other cellular sites, as well as the fact that cholesterol synthesis is not totally blocked by a single application of lovastatin, could provide cholesterol for lamellar body formation and thereby account for the modest levels of inhibition.
In summary, these studies provide the first direct evidence that de novo cutaneous sterol synthesis is required for the maintenance of barrier structure and function in the epidermis and suggests a crucial role for sterol synthesis in allowing for terrestrial existence.

\section{Acknowledgments}

We thank Pam Herranz for her excellent editorial assistance, Arthur Moser for valuable technical assistance, and Drs. Marvin Siperstein, Mary L. Williams, and Walter Holleran for helpful suggestions and advice.

This work was supported by the Medical Research Service, Veterans Administration, and National Institutes of Health grant AM-19098.

\section{References}

1. Blank, I. H. 1989. The protective role of the skin. In Dermatology in General Medicine. T. B. Fitzpatrick, A. Z. Eisen, K. Wolff, I. M. Freedberg, and K. F. Austin, editors. 3rd ed. McGraw-Hill Inc., New York. 337-342.

2. Elias, P. M., K. R. Feingold, G. K. Menon, S. Grayson, M. L. Williams, and G. Grubauer. 1987. The stratum corneum two-compartment model and its functional implications. In Skin Pharmacokinetics. B. Shrout and H. Schaefar, editors. S. Karger AG, Basel Switzerland. 1-8.

3. Elias, P. M., and K. R. Feingold. 1988. Lipid-related barriers and gradients in the epidermis. Ann. NY Acad. Sci. 548:4-13.

4. Turley, S. D., J. M. Andersen, and J. M. Dietschy. 1981. Rates of sterol synthesis and uptake in the major organs of the rat in vivo. $J$. Lipid Res. 22:551-569.

5. Feingold, K. R., M. H. Wiley, G. MacRae, S. R. Lear, A. H. Moser, G. Zsigmond, and M. D. Siperstein. 1983. De novo sterologenesis in the intact rat. Metabolism. 32:75-81.

6. Feingold, K. R., G. MacRae, A. H. Moser, J. Wu, and M. D. Siperstein. 1983. Differences in de novo cholesterol synthesis between the intact male and female rat. Endocrinology. 112:96-103.

7. Feingold, K. R., M. H. Wiley, A. H. Moser, D. T. Lau, S. R. Lear, and M. D. Siperstein. 1982. De novo sterologenesis in intact primates. J. Lab. Clin. Med. 100:405-410.

8. Feingold, K. R., B. E. Brown, S. R. Lear, A. H. Moser, and P. M. Elias. 1983. Localization of de novo sterologenesis in mammalian skin. J. Invest. Dermatol. 81:365-369.

9. Andersen, J. M., and J. M. Dietschy. 1977. Regulation of sterol synthesis in 15 tissues of the rat. II. Role of rat and human high and low density plasma lipoproteins and of rat chylomicron remnants. J. Biol. Chem. 252:3652-3657.

10. Brannan, P. G., J. L. Goldstein, and M. S. Brown. 1975. 3-Hydroxy-3-methylglutaryl coenzyme $A$ reductase activity in human hair roots. J. Lipid Res. 16:7-11.

11. Ponec, M., L. Havekes, J. Kempenaar, and B. J. Vermeet. 1983. Cultured human skin fibroblasts and keratinocytes: differences in the regulation of cholesterol synthesis. J. Invest. Dermatol. 81:125130.

12. Williams, M. L., S. L. Rutherford, A.-M. Mommaas-Kienhuis, S. Grayson, B. J. Vermeer, and P. M. Elias. 1987. Free sterol metabolism and LDL-receptor expression as differentiation markers in cultured human keratinocytes. J. Cell. Physiol. 132:428-440.

13. Mommaas-Kienhuis, A.-M., S. Grayson, M. C. Wijsman, B. J. Vermeer, and P. M. Elias. 1987. LDL receptor expression of keratinocytes in normal and psoriatic epidermis. J. Invest. Dermatol. 89:513517.

14. Menon, G. K., K. R. Feingold, A. H. Moser, B. E. Brown, and P. M. Elias. 1985. De novo sterologenesis in the skin. II. Regulation by cutaneous barrier requirements. J. Lipid Res. 26:418-427. 
15. Grubauer, G., K. R. Feingold, and P. M. Elias. 1987. The relationship of epiermal lipogenesis to cutaneous barrier function. $J$. Lipid Res. 28:746-752.

16. Feingold, K. R., B. E. Brown, S. R. Lear, A. H. Moser, and P. M. Elias. 1986. The effect of essential fatty acid deficiency on cutaneous sterol synthesis. J. Invest. Dermatol. 87:588-591.

17. Grubauer, G., P. M. Elias, and K. R. Feingold. 1989. Transepidermal water loss: the signal for recovery of barrier structure and function. J. Lipid Res. 30:323-334.

18. Alberts, A. W., J. Chen, G. Kuron, V. Hunt, J. Huff, C. Hoffman, et al. 1980. Mevinolin: a highly potent competitive inhibitor of hydroxy methylglutaryl-coenzyme A reductase and a cholesterol lowering agent. Proc. Natl. Acad. Sci. USA. 77:3957-3961.

19. Bittman, R., S. Clejan, and S. Rottem. 1983. Transbilayer distribution of sterols in mycoplasma membranes: a review. Yale J. Biol. Med. 56:397-403.

20. Elias, P. M., R. Orozco-Topate, M. A. Lampe, M. K. Nemanic, S. Grayson, M. L. Williams, and B. E. Brown. 1985. Methods in epidermal lipid research. In Methods in Skin Research. D. Skerrow and C. J. Skerrow, editors. John Wiley \& Sons, Inc., New York. $381-405$.

21. Elias, P. M., G. K. Menon, S. Grayson, and B. E. Brown. 1988. Membrane structural alterations in murine stratum corneum: relationships to the localization of polar lipids and phospholipases. $J$. Invest. Dermatol. 91:3-10.

22. Bligh, E. G., and W. J. Dyer. 1959. A rapid method of total lipid extraction and purification. Can. J. Biochem. Physiol. 37:911-917.

23. Ham, A. B. 1971. A new reagent for the determination of true cholesterol. Am. J. Med. Technol. 37:319-324.

24. Holleran, W. M., K. R. Feingold, M. Mao-Quiang, B. E. Brown, and P. M. Elias. 1990. Spingolipid synthesis in murine epidermis is regulated by permeability barrier requirements. Clin. Res. 38:635a. (Abstr.)

25. Grubauer, G., K. R. Feingold, and P. M. Elias. 1989. Lipid content and lipid type as determinants of the epidermal permeability barrier. J. Lipid Res. 30:89-96. 